\title{
Rol actual en la Argentina del médico especialista en diagnóstico por imágenes pediátrico (radiólogo pediatra) y su relación con las reflexiones de Richard Heller (EE.UU.)
}

\author{
Pediatric Imaging Specialist: current rol in Argentina and \\ its relationship with the reflections of Richard Heller
}

Dr. Luis Fernando Gentile ${ }^{a}$

http:/ /dx.doi.org/10.5546/aap.2014.474

La radiología pediátrica o diagnóstico por imágenes pediátrico tiene un papel indiscutible en el diagnóstico, seguimiento y tratamiento de las enfermedades pediátricas, como también en la atención al paciente y su familia. Esto último, como apoyo al clínico pediatra. ${ }^{1,2}$

Se ha publicado, en distintos artículos, que los radiólogos pediatras no son simplemente profesionales capacitados para interpretar o ejecutar estudios en niños. ${ }^{3,4}$ En sentido amplio, algunos radiólogos pediatras, con sensatez y espíritu analítico, comentan que hay dos características en la función del radiólogo pediatra: 1 ) una se refiere a su función específica y es la ejecución e informe de las imágenes con las distintas modalidades tecnológicas;

a. Área de Imágenes. Hospital de Niños Ricardo Gutiérrez. Director de la carrera de Diagnóstico por Imágenes Pediátrico. Universidad de Buenos Aires. Hospital de Niños Ricardo Gutiérrez. CABA.

\section{Correspondencia: \\ Dr. Luis \\ Fernando Gentile: luisfernandogentile1@ gmail.com}

Conflicto de intereses: Ninguno que declarar.

Recibido: 22-4-2014 Aceptado: 24-4-2014 y 2) otra no relacionada con la lectura o producción técnica de los estudios. El rol de "informante" se basa en el análisis y el informe de los exámenes de diagnóstico por imágenes. Este es el valor proporcionado por la "lectura de placas" de cualquier modalidad que utilicemos. Evaluamos al buen "informante" como aquel que posee velocidad de interpretación (determinada por el tiempo utilizado para realizar el informe) y en la precisión o exactitud de las descripciones. El punto 2 o segunda función del radiólogo se refiere a otras tareas que cumple, que incluyen el tiempo empleado en las consultas telefónicas o personales con médicos clínicos, en el diálogo con los pacientes y sus familiares en estudios simples o contrastados de mayor complejidad, en la asistencia a conferencias y congresos de capacitación y en la revisión de los algoritmos en reuniones de consenso con otras especialidades. ${ }^{5,6}$

Son importantes las funciones del radiólogo no relacionadas con su formación técnica y analítica de las imágenes, tales como evaluar que el estudio se ajuste a la indicación, supervisarlo y comunicarse telefónica o personalmente con el médico mandante $y$, según el caso, con los pacientes. 7,8

La radiología pediátrica es una subespecialidad que siempre ejerció un valor agregado fuera del trabajo específico. Históricamente, uno de los objetivos es limitar la cantidad de estudios por imágenes realizados en niños, en particular por la exposición a la radiación. ${ }^{9}$

En cuanto a la función como interconsultores, históricamente, los radiólogos pediátricos mantuvieron una estrecha relación con sus pares clínicos. El diálogo personal ofrece a los clínicos la posibilidad de conocer la opinión exacta del radiólogo, en especial cuando se le brinda el cuadro clínico completo, que nunca se incluye en una simple solicitud de un examen. También permite al radiólogo realizar un informe más preciso al contar con el contexto clínico apropiado. Por último, se obtiene un beneficio educativo tanto para aquellos médicos de radiología y pediatría que se están formando como para los profesionales con mayor experiencia. 
En nuestra experiencia, con una residencia oficial de radiología pediátrica muy joven en la Argentina, lo expresado es de mucho valor para el crecimiento profesional y consecuente beneficio de los pacientes. ${ }^{10}$

Es probable que gran parte de este intercambio entre radiólogos y clínicos se haya perdido en algunos sectores, de modo que los clínicos o especialistas pediátricos simplemente solicitan los estudios para sus pacientes. De acuerdo con este último sistema, los radiólogos se centran en la interpretación, es decir, informan los estudios de manera precisa y puntual, pero cediendo a los médicos clínicos el control sobre la solicitud de estudios. Estos últimos asumen un papel de profesionales experimentados, capaces de determinar qué estudio de imágenes satisface más sus necesidades, independientemente de su capacitación o experiencia real en este campo. ${ }^{11}$

El radiólogo pediatra tiene un objetivo básico: limitar la cantidad y el alcance de los estudios de diagnóstico por imágenes en los niños. En pediatría, esto se lleva a cabo para limitar los efectos nocivos de la radiación, siguiendo el principio de ALARA (as low as reasonably achievable) [tan baja como sea razonablemente posible] y con la campaña Image Gently (Diagnóstico por Imágenes con Cuidado). Aunque incluso los estudios con radiación no ionizante se limitan en número y alcance, más allá de la radiación, también existe el problema de la anestesia en algunos exámenes, $\mathrm{y}$, aunque en los hospitales de niños esta última es segura y frecuente, no se debe llevar a cabo sin una indicación firme.

Cuando la TC es la mejor opción, se trata de focalizar el estudio. Por ejemplo, en el hospital donde ejerce el Dr. Heller, todas las TC pediátricas se realizan en una sola etapa, a menos que se decida lo contrario tras discutir con un radiólogo pediátrico que esté presente en el estudio. En algunas ocasiones, ciertos médicos externos solicitan una TC pediátrica "con y sin contraste", pero a los técnicos del hospital derivado se los ha capacitado para que señalen este tipo de estudios y consulten a un radiólogo pediátrico antes de realizarlo. ${ }^{12}$ En el Hospital de Niños Ricardo Gutiérrez de Buenos Aires, los estudios de mayor complejidad (RM o TC) solicitados por clínicos o especialistas del hospital requieren de una firma autorizante previa por parte de un médico radiólogo pediatra del staff del servicio para su realización. Esto ayudó desde el año 2002 a una mejor prescripción y racionalización de los exámenes.

Los médicos mandantes constituyen el pilar de los departamentos de radiología y de ellos depende el flujo de producción. En las últimas jornadas del Hospital de Niños Ricardo Gutiérrez del año 2012, en la sesión de Diagnóstico por Imágenes, se expuso la problemática de la exacta indicación y justificación de estudios, y se sintetizó la dinámica en un gráfico llamado "circuito radiológico". Además, el habitual desconocimiento de la clínica del paciente por parte del radiólogo fue señalado como una barrera o valla que reduce la eficacia del examen. ${ }^{13}$

En conclusión, debido a la necesidad constante de optimizar pedidos y justificar el uso de los estudios, deberá priorizarse el diálogo fluido y constante entre los integrantes del equipo de atención para intercambiar conocimientos y proponer algoritmos para un mejor uso de las imágenes. ${ }^{14}$

\section{REFERENCIAS}

1. Japsen B. Small-picture approach flips medical economics. The New York Times 2012. Pág.D1.

2. Centers for Medicare and Medicaid Services. Baltimore: Federal Government US; 2012. Disponible en: http:/ / www. cms.gov. [Consulta: 10 de diciembre de 2012].

3. Smith-Bindman R, Miglioretti DL, Larson EB. Rising use of diagnostic medical imaging in a large integrated health system. Health Aff (Millwood) 2008;27(6):1491-502.

4. Medical Imaging \& Technology Alliance. Imaging today: medical imaging trends in Medicare. September 20, 2012. Virginia: National Electrical Manufacturers Association; 2012. Disponible en: http://www.medicalimaging.org/ wp-content/uploads/2012/09/Medicare-2011-Data-MITA-Report-Final-9.20.2012.pdf. [Consulta: 10 de diciembre de 2012].

5. Maynard CD. Radiologists: physicians or expert image interpreters? Radiology 2008;248(2):333-6.

6. Slovis TL. The easier path: a blueprint for failure. Pediatr Radiol 2007;37(5):415-6.

7. Slovis TL. The path less travelled-a road to success. Pediatr Radiol 2007;37(6):513-4.

8. Blackmore CC, Mecklenburg RS. Taking charge of imaging: implementing a utilization program. Appl Radiol 2012;41(78):18-23.

9. Lehnert BE, Bree RL. Analysis of appropriateness of outpatient CT and MRI referred from primary care clinics at an academic medical center: how critical is the need for improved decision support? J Am Coll Radiol 2010;7(3):192-7.

10. Allen B Jr, Levin DC, Brant-Zawadzki M, Lexa FJ, et al. ACR white paper: strategies for radiologists in the era of health care reform and accountable care organizations: a report from the ACR Future Trends Committee. J Am Coll Radiol 2011;8(5):309-17.

11. Regents Health Resources. Hospital-based versus freestanding outpatient imaging services. Providence: Radiology Business Journal; 2011. Disponible en: http:/ /www. radiologybusiness.com/topics/business/hospital-basedversus-freestanding-outpatient-imaging-services]. [Consulta: 10 de diciembre de 2012.

12. Heller RE $3^{\text {rd }}$. The 21st-century radiology department: lessons from the pediatric section. Pediatr Radiol 2013;43(5):536-8.

13. Vásquez Millán S. Diagnóstico por imágenes en pediatría: ¿cómo, cuándo y para qué? XXX Jornadas del Hospital de Niños Ricardo Gutiérrez, 2013, 15 al 18 de octubre, Buenos Aires; Hospital de Niños Ricardo Gutiérrez.

14. Moguillansky S. El radiólogo pediatra en Latinoamérica. En: López Marure E, ed. Radiología pediátrica para pediatras. Ciudad de Buenos Aires: Journal; 2009. Pág.19. 